\title{
Treatment of $B-R A F$ mutant human tumor cells with a MEK inhibitor requires Bim and is enhanced by a $\mathrm{BH} 3$ mimetic
}

\author{
Mark S. Cragg, ${ }^{1,2}$ Elisa S. Jansen, ${ }^{1}$ Michele Cook, ${ }^{1}$ Claire Harris, ${ }^{2}$ \\ Andreas Strasser, ${ }^{1}$ and Clare L. Scott ${ }^{1}$ \\ ${ }^{1}$ The Walter and Eliza Hall Institute of Medical Research (WEHI), Melbourne, Victoria, Australia. ${ }^{2}$ Cancer Sciences Division, \\ Southampton University, Southampton, United Kingdom.
}

\begin{abstract}
$B-R A F$ is frequently mutated in solid tumors, resulting in activation of the MEK/ERK signaling pathway and ultimately tumor cell growth and survival. MEK inhibition in these cells results in cell cycle arrest and cytostasis. Here, we have shown that MEK inhibition also triggers limited apoptosis of human tumor cell lines with $B-R A F$ mutations and that this effect was dependent on upregulation and dephosphorylation of the proapoptotic, Bcl-2 homology 3-only (BH3-only) Bcl-2 family member Bim. However, upregulation of Bim was insufficient for extensive apoptosis and was countered by overexpression of $\mathrm{Bcl}-2$. To overcome apoptotic resistance, we treated the $B-R A F$ mutant cells both with MEK inhibitors and with the BH3 mimetic ABT-737, resulting in profound synergism and extensive tumor cell death. This treatment was successful because of both efficient antagonism of the prosurvival Bcl-2 family member Mcl-1 by Bim and inhibition of Bcl-2 and Bcl- $x_{L}$ by ABT737. Critically, addition of ABT-737 converted the predominantly cytostatic effect of MEK inhibition to a cytotoxic effect, causing long-term tumor regression in mice xenografted with human tumor cell lines. Thus, the therapeutic efficacy of MEK inhibition requires concurrent unleashing of apoptosis by a $\mathrm{BH} 3$ mimetic and represents a potent combination treatment for tumors harboring $B-R A F$ mutations.
\end{abstract}

\section{Introduction}

The Ras/Raf/MEK/ERK signaling pathway regulates cellular proliferation, differentiation, and survival (1). Aberrant activation of this pathway, often caused by activating mutations in the composite enzymes, occurs in many tumors $(2,3)$. In human cancer, mutations in $R A F$ (mainly $B-R A F$ ) occur in approximately $60 \%$ of melanomas (3) and with lower frequency in papillary thyroid cancers (4), colorectal carcinomas $(3,5,6)$, and lung cancers (3). This spectrum of malignancies is similar to that observed with $R A S$ mutations, found in about $15 \%-30 \%$ of human cancers overall (3, $7,8)$, which indicates that dysregulation of the Ras/Raf/MEK/ERK pathway may be central to the genesis of these malignancies $(2,3)$. It was recently shown that $B-R A F$ mutant cells are considerably more sensitive to MEK inhibition than are either RAS mutant or $B-R A F / R A S$ WT cells (9). In the B-RAF mutant cells, MEK inhibition elicited potent cell cycle arrest and also apoptosis in some cases, but the mechanisms for cell killing were not examined.

Tumor cell apoptosis can occur via extrinsic (death receptor) or intrinsic (mitochondrial) cell death pathways (10). Intrinsic apoptosis is regulated by the $\mathrm{Bcl}-2$ family proteins, consisting of 3 subgroups: the prosurvival members, such as Bcl-2 or Mcl-1, the proapoptotic Bax/Bak subgroup, and the proapoptotic Bcl-2 homology 3-only (BH3-only) proteins. Apoptotic stimuli trigger activation of specific $\mathrm{BH} 3$-only proteins, which then engage the prosurvival Bcl-2 family members and liberate the downstream effectors, Bax and Bak, to elicit mitochondrial outer membrane permeabilization, unleashing the caspase cascade and culminating

Nonstandard abbreviations used: $\mathrm{BH} 3, \mathrm{Bcl}-2$ homology 3 ; ILS, increase in lifespan; KD, knockdown; PR, partial response.

Conflict of interest: The authors have declared that no conflict of interest exists. Citation for this article: J. Clin. Invest. 118:3651-3659 (2008). doi:10.1172/JCI35437. in cell demolition. Based on discoveries with other kinase inhibitors (11-14), we hypothesized that MEK inhibitors would kill $B$ - $R A F$ mutant tumor cells by upregulating $\mathrm{BH} 3$-only proteins.

Here we present data demonstrating that MEK inhibitors kill $B-R A F$ mutant tumor cells by upregulating the expression of the proapoptotic BH3-only protein Bim and present evidence that MEK inhibitors synergize with the $\mathrm{BH} 3$ mimetic ABT-737 to cause tumor cell apoptosis. Finally, we provide what we believe to be the first evidence that the combination of MEK inhibition and ABT737 induces potent antitumor effects in vivo.

\section{Results}

MEK inbibition caused growth arrest and apoptosis in B-RAF mutant tumor cells. Initial studies confirmed the previous observation (9) that the MEK inhibitor UO126 potently inhibited proliferation of the B-RAF mutant (V600E) tumor cell lines Colo205 and SkMel-28, but had little impact on the WT B-RAF PC3 tumor cell line (Figure $1 A)$. In addition, we found that following $G_{1}$ cell cycle arrest, a sizeable proportion of Colo205 and SkMel-28 cells underwent apoptosis, as indicated by sub-G $\mathrm{G}_{1}$ DNA content (Figure 1, A and B) as well as cleavage of PARP and caspase-3 (Figure 1C). The extent of tumor cell killing depended on the dose of the MEK inhibitor, correlated with reduced phosphorylation of ERK1/2 (Figure 1C), and was inhibited by the broad-spectrum caspase inhibitor QVD$\mathrm{OPH}$ and by $\mathrm{Bcl}-2$ overexpression (Figure 1D). These findings were reproduced with an independent MEK inhibitor, PD98059, although it was less potent than UO126 (Figure 1C and data not shown). These results show that MEK inhibition caused cell cycle arrest and Bcl-2-regulated apoptosis (also called mitochondrial or intrinsic apoptosis) in $B-R A F$ mutant tumor cells.

$M E K$ inhibition caused the induction of Bim in B-RAF mutant tumor cells. Because MEK inhibition-induced apoptosis of Colo205 cells 

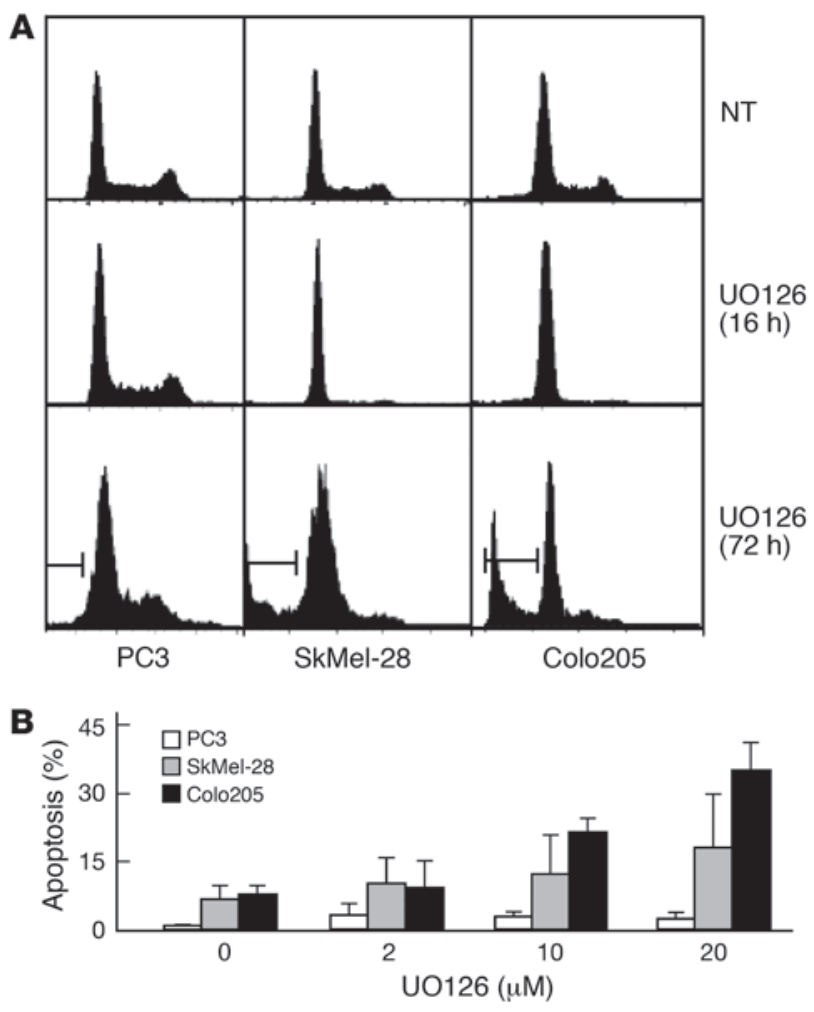

C

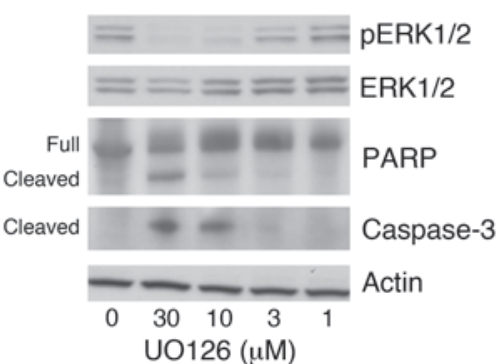

D

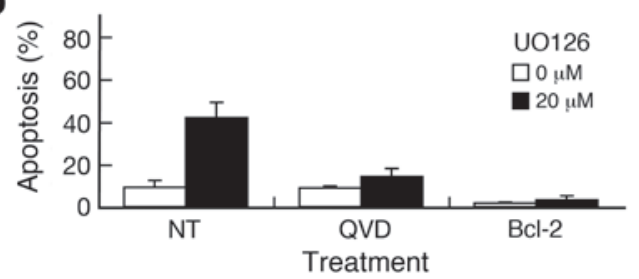

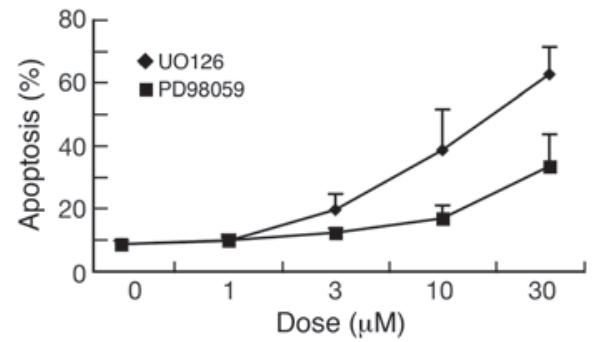

E

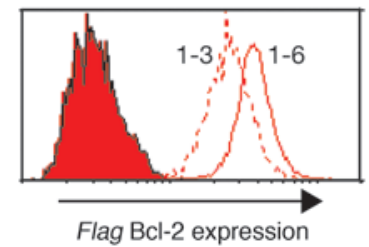

\section{Figure 1}

MEK inhibition causes growth arrest and apoptosis in $B-R A F$ mutant tumor cells. (A and $\mathrm{B}$ ) $B-R A F \mathrm{WT}$ (PC3) or mutant (SkMel-28 and Colo205) cells were not treated (NT) or were treated for 16 or $72 \mathrm{~h}$ with the MEK inhibitor U0126 (20 $\mu \mathrm{M}$ unless otherwise indicated), and DNA content was determined by FACS analysis. (A) Illustrative FACS plots show untreated cells, cells undergoing $\mathrm{G}_{1}$ arrest and apoptosis after 16 and $72 \mathrm{~h}$, respectively, of U0126 treatment. Bars denote sub-G ${ }_{1}$ DNA content. (B) Percent cells with sub-G 1 DNA content at 72 h. (C) Colo205 cells were treated for $48 \mathrm{~h}$ with the indicated doses of UO126 or PD98059. Cells were analyzed by Western blotting for phosphorylated ERK ( $p E R K 1 / 2)$, total ERK, PARP, cleaved caspase- 3 , and $\beta$-actin as loading control and were also assessed for cell death (shown at right). For $\mathbf{B}$ and $\mathbf{C}$, data are mean $\pm \mathrm{SD}$ of 3 independent experiments. (D) Colo205 cells were not treated or were incubated with $25 \mu \mathrm{M}$ QVD-OPH (QVD) for 30 min prior to addition of $20 \mu \mathrm{M}$ UO126 and assessed after $48 \mathrm{~h}$ for cell death and cell cycle. Colo205 cells overexpressing FLAG-Bcl-2 were assessed in the same manner. Data are mean \pm SD of 3 independent experiments using both FLAG-Bcl-2 clones. (E) Bcl-2 expression levels for clones $1-3$ and 1-6. Filled histogram represents staining with a control antibody. was blocked by Bcl-2 overexpression, we examined the expression of other Bcl-2 family members. Treatment with UO126 caused a rapid and sustained induction of Bim in Colo205 cells, but not in PC3 cells (Figure 2A). Of the known isoforms of Bim that are generated by alternative splicing (15), Bim $\mathrm{EL}_{\mathrm{L}}$ was the most prominently expressed, but $\mathrm{Bim}_{\mathrm{L}}$ was also detected (Figure $2 \mathrm{~A}$ ). The extent of Bim induction in Colo205 cells was dose dependent and correlated with the extent of ERK1/2 dephosphorylation (Figure 2B). No significant changes were observed in other $\mathrm{BH} 3$-only proteins (e.g., $\mathrm{Bad}$, Bid, or Puma), proapoptotic Bax and Bak, or the prosurvival proteins $\mathrm{Bcl}-2$, Bcl-w, Bcl- $\mathrm{x}_{\mathrm{L}}$, and Mcl-1 (Figure 2, A and B); prosurvival protein $\mathrm{A} 1$ was below the level of detection (data not shown). These results show that MEK inhibition caused specific induction of Bim in B-RAF mutant tumor cells.
MEK inbibition caused dephosphorylation of Bim in B-RAF mutant Colo205 cells. Activation of Bim often involves its dephosphorylation, resulting in a reduction in apparent molecular weight on SDSPAGE analysis $(16,17)$. Such a change in BimeL was apparent after treatment of Colo205 cells with UO126 (Figure 2, A and B), and a change in Bim phosphorylation was supported by phosphatase treatment of cell lysates (Figure 2C). In contrast, similar analysis of PC3 cells revealed very little difference in the migration of Bim $\mathrm{EL}$ after MEK inhibition. Using phosphorylated Bad-specific antibodies, it was apparent that neither residue 112 nor residue 136 of Bad were substantially dephosphorylated in Colo205 cells after MEK inhibition (Figure 2D). These data indicate that Bim was constitutively phosphorylated in $B-R A F$ mutant tumor cells and that MEK inhibition caused its specific dephosphorylation. 


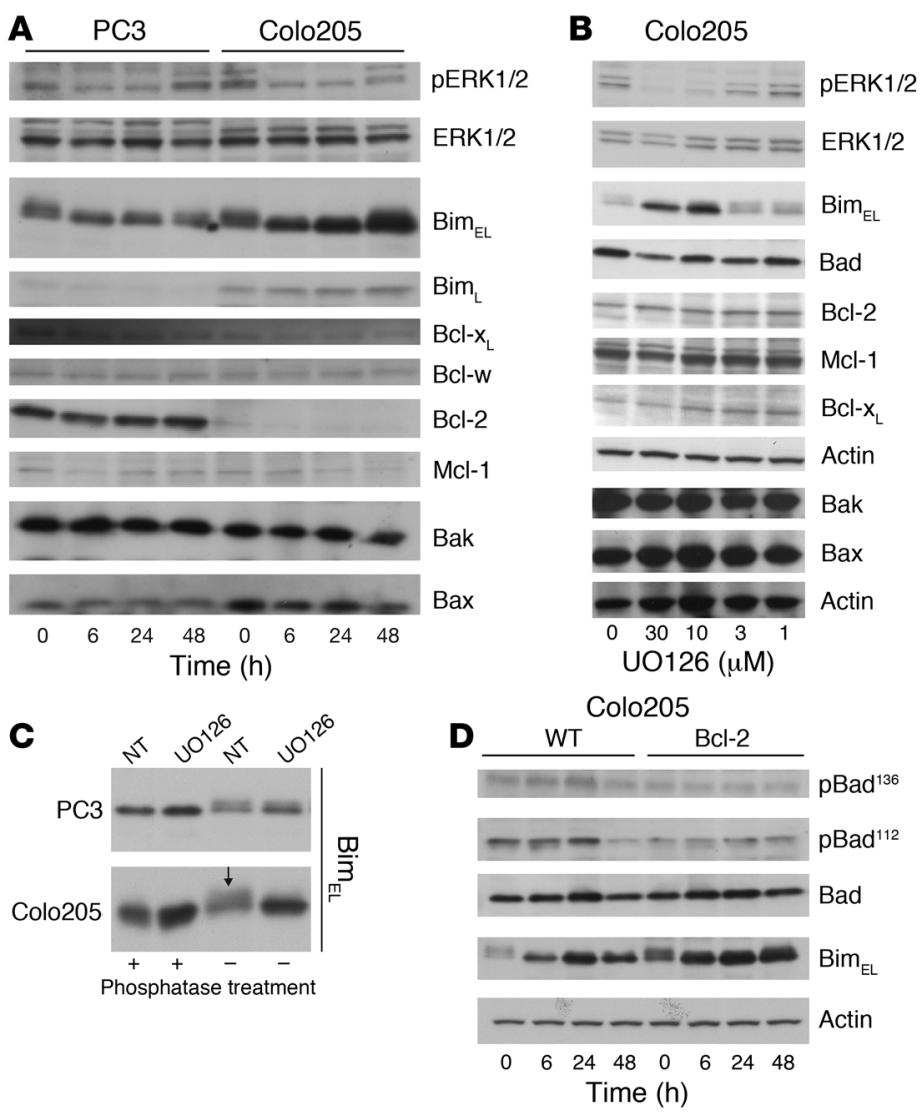

\section{Figure 2}

Effect of MEK inhibition on the expression and phosphorylation of $\mathrm{BH} 3-o n l y$ proteins and prosurvival $\mathrm{Bcl}-2$ family members. (A) $B-R A F$ WT PC3 cells and $B-R A F$ mutated Colo205 cells were not treated or were treated for 6,24 , or $48 \mathrm{~h}$ with $20 \mu \mathrm{M}$ UO126 and assessed by Western blotting for expression of the indicated proteins. (B) Colo205 cells were treated for $48 \mathrm{~h}$ with UO126 and assessed by Western blotting for the indicated proteins. The lysates examined here were the same as those probed in Figure $1 \mathrm{C}$, and the blots shown for phosphorylated ERK, total ERK, and actin are identical, included to allow for direct comparison between loss of ERK phosphorylation and change in apoptosis proteins. Western blot analysis of Bax and Bak levels was performed with new lysates from identically treated cells, with equal loading demonstrated by probing for $\beta$-actin. (C) PC3 and Colo205 cells were not treated or were treated for $18 \mathrm{~h}$ with $20 \mu \mathrm{M}$ U0126, harvested, and lysed. Lysates were not treated or were treated with $\lambda$ phosphatase, and the migration of Bim was assessed by Western blotting. In healthy Colo205 cells, Bim $\mathrm{EL}_{\mathrm{L}}$ appeared as a broad band (arrow). Treatment with $\lambda$ phosphatase produced a single band of apparent lower molecular weight similar to that after treatment with UO126. (D) Control and Bcl-2-overexpressing Colo205 cells were not treated or were treated for 6,24 , or $48 \mathrm{~h}$ with $20 \mu \mathrm{M}$ U0126 and assessed by Western blotting. Data are representative of 3 independent experiments.
Bim was essential for MEK inhibition-induced apoptosis and loss of clonogenicity in B-RAF mutant tumor cells. To examine the role of Bim in MEK inhibition-induced cell killing of $B-R A F$ mutant cells, we generated subclones of Colo205 cells in which RNAi stably repressed Bim expression (Figure 3A and Supplemental Figure 1A; supplemental material available online with this article; doi:10.1172/ JCI35437DS1). These Bim knockdown (KD) cells were protected from UO126-induced killing (Figure 3B and Supplemental Figure $1 \mathrm{~A})$, although not as potently as those overexpressing Bcl-2, most likely because of incomplete KD of Bim (Figure 3A). Importantly, the Bim KD and Bcl-2-overexpressing cells underwent dephosphorylation of ERK1/2 and cell cycle arrest in response to UO126 treatment, demonstrating that they still responded to MEK inhibition (Figure $3 \mathrm{~A}$ and data not shown). Bim KD and Bcl-2 overexpression had similar effects on the response to MEK inhibition in SkMel-28 melanoma cells, an independent $B-R A F$ mutant tumor cell line (Supplemental Figure 1B). Treatment with UO126 for $24 \mathrm{~h}$ caused an approximately 10 -fold reduction in colony formation of parental Colo205 cells, which was blocked by both Bim KD and Bcl-2 overexpression (Figure 3C). However, after 48 h of UO126 treatment, only $\mathrm{Bcl}-2$ overexpression provided protection against loss of clonogenicity, again most likely because of incomplete $\mathrm{KD}$ of Bim with longerterm MEK inhibition (Figure 3A). Collectively, these results demonstrate that Bim is essential for MEK inhibition-induced apoptosis and loss of clonogenicity of $B-R A F$ mutant tumor cells.

$M E K$ inhibition caused induction and dephosphorylation of Bim in a range of B-RAF mutant tumor cell lines. Next, we extended our analysis to 3 additional $B-R A F$ mutant tumor cell lines: SkMel-28, MM200-1, and Mel-RMU. In all of these melanoma cell lines, profound dephosphorylation of ERK1/2 and robust induction of Bim were observed after MEK inhibition (Figure 4A). Similar to what we observed in Colo2 25 cells, analysis of the mobility of Bim on SDSPAGE and treatment of lysates with $\lambda$ phosphatase indicated that MEK inhibition caused dephosphorylation of Bim in SkMel-28 cells (Figure 4B). These data cement the notion that MEK inhibition causes Bim upregulation in $B-R A F$ mutant tumor cells.

Induction of apoptosis requires efficient antagonism of all prosurvival Bcl-2 family members present in a given cell by BH3-only proteins (18). Bim, unlike more selective $\mathrm{BH} 3$-only proteins (such as Bad), can bind with high affinity to all prosurvival Bcl-2 family members $(19,20)$. Therefore, survival of MEK inhibitor-treated $B$ - $R A F$ mutant tumor cells, despite robust Bim induction, may be a consequence of high levels of prosurvival Bcl-2-like proteins and/ or very low levels of other $\mathrm{BH} 3$-only proteins. We therefore performed a comprehensive $\mathrm{BH} 3$-only protein and prosurvival $\mathrm{Bcl}-2-$ like protein analysis in these cells. This revealed that the SkMel-28, MM200-1, and Mel-RMU cell lines all contained lower basal levels of Bim and higher levels of phosphorylated ERK1/2, and the SkMel-28 and MM200-1 lines demonstrated higher levels of Bcl-2, than did the more sensitive Colo205 cells (Figure 4C). In contrast, they contained comparable levels of other Bcl-2 family members, including Bmf, Puma, Bad, Bax, Bak, and Mcl-1 (Figure 4C). Thus, the relative levels of Bim and Bcl-2 may contribute to the observed differences in sensitivity of the different $B-R A F$ mutant cells to MEK inhibition-induced apoptosis.

The BH3 mimetic ABT-737 synergized with MEK inbibition in the killing of $B$-RAF mutant tumor cells. Since low levels of BH3-only proteins and/ or high levels of Bcl-2-like prosurvival proteins may limit the cyto- 

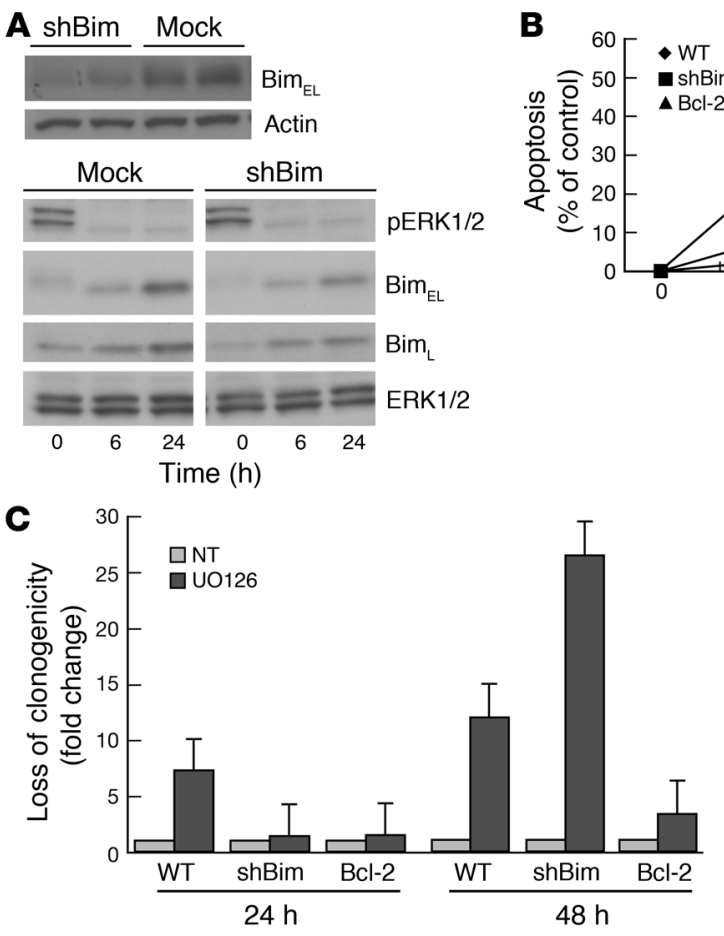

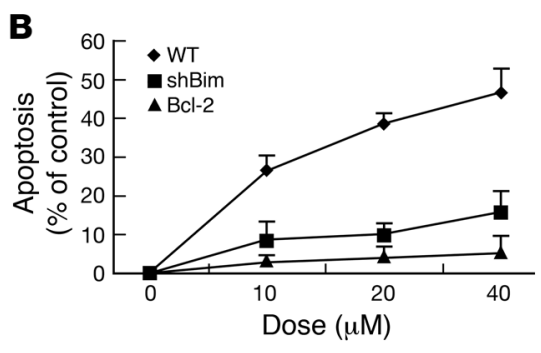

\section{Figure 3}

MEK inhibition-induced apoptosis of $B-R A F$ mutant tumor cells can be inhibited by Bim KD or Bcl-2 overexpression. (A) Top: Western blot analysis documents the levels of Bim and $\beta$-actin (loading control) expression in parental and 2 independent RNAi Bim KD subclones of Colo205 cells. Bottom: Parental and RNAi Bim KD subclone 18 Colo205 cells were not treated or were treated for 6 or $24 \mathrm{~h}$ with $20 \mu \mathrm{M}$ U0126 and analyzed by Western blotting for their levels of Bim. (B) Parental, Bim RNAi KD, and Bcl-2-overexpressing clones of Colo205 cells were treated for $48 \mathrm{~h}$ with 0-40 $\mu \mathrm{M}$ UO126 as indicated, and cell survival was examined by FACS analysis. Data (mean \pm SD of 3 or 4 independent clones) indicate percent cell death relative to untreated cells. (C) Clonogenic survival assays of parental, Bim RNAi KD, and Bcl-2-overexpressing clones of Colo205 cells without treatment or after 24 or $48 \mathrm{~h}$ of treatment with $20 \mu \mathrm{M}$ UO126. Data are mean \pm SD of 3 independent experiments. toxic activity of MEK inhibition (Figure 3 and Figure 4C), we sought to determine whether a BH3 mimetic, such as ABT-737 (21), could enhance killing of $B-R A F$ mutant tumor cells. The MEK inhibitor sensitivity of a tumor cell line with a sensitive profile (i.e., Colo205; low Bcl-2 and high Bim) was further enhanced by the addition of ABT-737 in a dose-dependent manner, resulting in far greater killing than achieved with either drug alone (Figure 5, A and B). Apoptosis induced by this drug combination was blocked by QVD-OPH, which indicates that it was caspase dependent (data not shown). Because Bim KD and Bcl-2 overexpression rendered Colo205 cells resistant to MEK inhibition, we examined whether these cells could be resensitized by the addition of ABT-737. Treatment with ABT-737 or UO126 alone produced modest effects, but in combination, these drugs achieved killing of large fractions of Bim KD and even Bcl-2overexpressing Colo205 cells (Figure 5C). Perhaps most striking was the ability of $1 \mu \mathrm{M}$ ABT-737 to resensitize $\mathrm{Bcl}$-2-overexpressing Colo205 cells, which were completely refractory to MEK inhibition alone (Figure 5C) and also resistant to etoposide-induced apoptosis (Supplemental Figure 2). In support of our hypothesis that SkMel-28 and MM200-1 tumor cells are relatively resistant to MEK inhibition because they express comparatively low levels of Bim and high levels of Bcl-2, treatment with the combination of UO126 and ABT-737 resulted in substantially more apoptosis compared with treatment with either drug alone (Figure 5D and Supplemental Figure 3). In contrast, combination of the same concentrations of UO126 and ABT-737 did not cooperate in killing $2 B-R A F$ WT tumor cell lines (Figure 5D). Finally, combinations of UO126 and ABT-737 overcame the suppression of apoptosis achieved in SkMel-28 cells by Bim KD and Bcl-2 overexpression (data not shown). Collectively, these results demonstrate that ABT-737 and MEK inhibition synergized in killing $B-R A F$ mutant tumor cells.

Addition of ABT-737 increased the extent of Bim complexed with Mcl-1. Because apoptosis induction requires antagonism of all prosurvival molecules expressed in a given cell by $\mathrm{BH} 3$-only proteins (18), we hypothesized that the synergistic effects of UO126 and ABT737 may result from the ability of ABT-737 to bind Bcl-2, Bcl-w, and $\mathrm{Bcl}-\mathrm{x}_{\mathrm{L}}$, thereby releasing Bim and allowing it to bind to $\mathrm{Mcl}-1$ and A1. To investigate this, we immunoprecipitated Bim from Colo205 cells, followed by Western blotting for Bcl- $\mathrm{x}_{\mathrm{L}}$ and $\mathrm{Mcl}-1$ to determine the prosurvival binding partners of Bim in the presence of UO126 with or without addition of ABT-737. Treatment with ABT-737 resulted in a decrease of Bcl- $\mathrm{x}_{\mathrm{L}}$ but a concomitant increase in Mcl-1 complexed to Bim (Figure 5, E and F). Similar results were obtained with Colo205 cells overexpressing Bcl-2 (Figure $5 \mathrm{E}$ ) with or without concomitant MEK inhibition and with Colo205 cells grown in nude mice as subcutaneous tumors, then treated in vivo with ABT-737 (Figure 5G). These results showed that treatment with ABT-737 promoted increased association of Bim with Mcl-1 by causing release of Bim from Bcl-2 and $\mathrm{Bcl}-\mathrm{x}_{\mathrm{L}}$.

$M E K$ inhibition and ABT-737 synergized to enhance survival of mice bearing B-RAF mutant tumors. Next we examined whether ABT-737 cooperates with MEK inhibition in the treatment of $B-R A F$ mutant tumors in vivo. We used PD0325901, which has a much higher affinity for MEK and enhanced efficacy in vivo than does UO126 (22). As anticipated, in vitro treatment of SkMel-28 or Colo205 tumor cells with $50 \mathrm{nM}$ PD0325901 resulted in potent inhibition of ERK1/2, robust induction of Bim (Supplemental Figure 4), and extensive apoptosis (data not shown). In mice bearing SkMel-28 tumors, after $48 \mathrm{~h}$ of in vivo treatment with either $3 \mathrm{mg} / \mathrm{kg}$ PD0325901 or with the combination of $3 \mathrm{mg} / \mathrm{kg}$ PD0325901 and $75 \mathrm{mg} / \mathrm{kg} \mathrm{ABT-737,} \mathrm{robust}$ induction of Bim was seen in the tumor cells (Figure 6A). Tumorbearing mice were treated for $10 \mathrm{~d}$ with the respective regimen, and no significant clinical toxicity was observed as evidenced by stable weight, normal behavior and hematologic analysis, although platelet levels fell initially, as expected, in the ABT-737 groups (Supplemental Figure 5 and ref. 23). Administration of PD0325901 to SkMel-28 tumor-bearing mice inhibited tumor growth, caused transient, partial tumor regression - referred to herein as partial response 
A
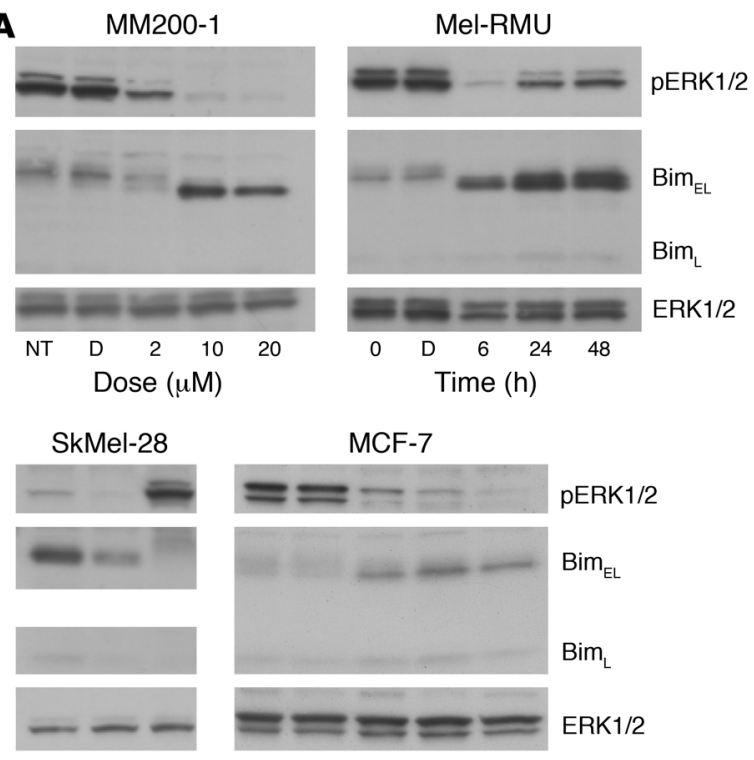

$48 \quad 24 \quad 0$

Time (h)

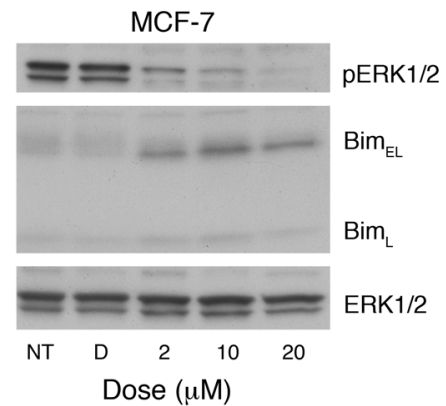

B

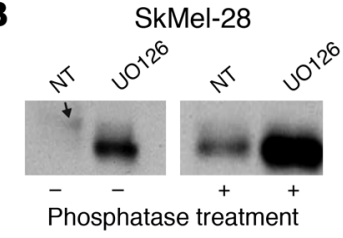

C

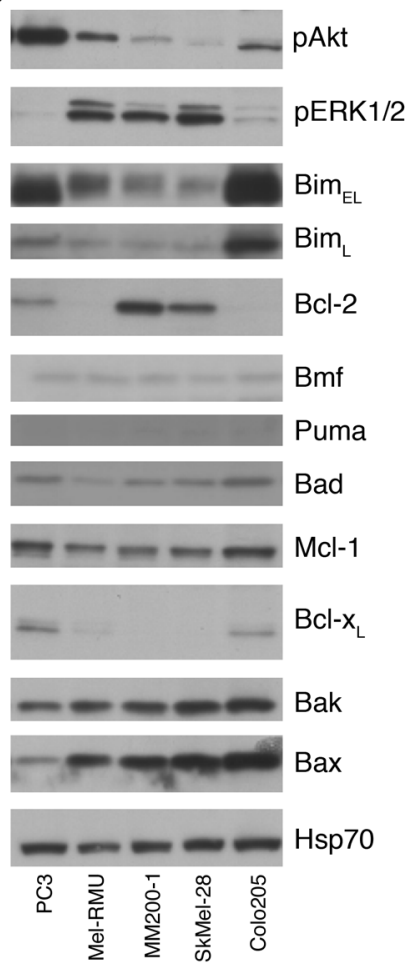

\section{Figure 4}

MEK inhibition causes induction and dephosphorylation of Bim in a range of $B-R A F$ mutant tumor cells. (A) MM200-1, SkMel-28, Mel-RMU, and MCF-7 tumor-derived cell lines were not treated, were treated with $20 \mu \mathrm{M}$ UO126 for the indicated time points, or were treated with the indicated concentrations of UO126 for $18 \mathrm{~h}$, and were assessed for levels of Bim, phosphorylated ERK1/2, and total ERK $1 / 2$ by Western blotting. D, DMSO control. (B) SkMel-28 cells were not treated or were treated for $18 \mathrm{~h}$ with $20 \mu \mathrm{M}$ U0126, harvested, and lysed. Lysates were not treated or were treated with $\lambda$ phosphatase and then assessed by Western blotting for the migration of Bim on SDS-PAGE. Arrow indicates the faint diffuse band of Bim present in untreated healthy cells. (C) Untreated PC3, MM200-1, SkMel-28, Mel-RMU, and Colo205 cells were assessed by Western blotting for the indicated apoptosis-related proteins, all on the same membranes to allow direct comparisons.
(PR), defined by tumor shrinkage of at least $50 \%$, but less than $100 \%$ - in 2 of 10 mice for $2 \mathrm{~d}$, and prevented tumor progression for about $1 \mathrm{wk}$ after treatment had finished, whereas ABT-737 had no effect on its own (Figure 6, B and C). Remarkably, the combination of PD0325901 and ABT-737 resulted in profound inhibition of tumor growth, with tumor regression (complete response, i.e., $100 \%$ reduction, in 1 recipient mouse and $P R$ in all 12 remaining mice) for a median of $7 \mathrm{~d}$ and a delay in tumor progression that persisted more than 9 wk after treatment stopped (median survival, $83 \mathrm{~d}$ for the combination versus $45 \mathrm{~d}$ for PD0325901 alone, $P<0.0007$; increase in lifespan [ILS], 184\% versus PD0325901 alone; Figure 6, B and C; and ref. 24). ABT-737 and PD0325901 also cooperated in the treatment of nude mice bearing Colo205 tumors (median survival, $30 \mathrm{~d}$ for the combination versus $20 \mathrm{~d}$ for PD0325901 alone; ILS, $150 \%$ versus PD0325901 alone; $P<0.005$; Supplemental Figure 6). Furthermore, upon reaching the maximal tumor volume (predetermined according to animal ethics guidelines; see Methods), in retreatment studies with the same 10-d regimen PD0325901 alone - and, more strikingly, the combination of PD0325901 and ABT737 - again elicited substantial tumor regression. Treatment with $3 \mathrm{mg} / \mathrm{kg}$ PD0325901 in SkMel-28 tumor-burdened mice resulted in PR in 0 of 2 mice compared with PR in 3 of 3 mice retreated with PD0325901 plus ABT-737; Colo205 tumor-burdened mice retreated with PD0325901 underwent PR in 1 of 3 mice, compared with PR in 2 of 2 mice retreated with PD0325901 plus ABT-737. This finding indicates that tumors remained susceptible to target inhibition at the time of relapse (Supplemental Figure 7). Our results show that MEK inhibition and ABT-737 can synergize to produce impressive in vivo antitumor efficacy in mice bearing $B-R A F$ mutant tumors.

\section{Discussion}

Our results show that 3 well-characterized MEK inhibitors, UO126, PD98059, and PD0325901, triggered apoptosis in B-RAF mutant, but not $B-R A F$ WT, tumor cells. Although MEK inhibition-induced $\mathrm{G}_{1}$ cell cycle arrest was similar in all $4 B-R A F$ mutant tumor cell lines examined, the extent of apoptosis was much more varied, in agreement with previously published work (9). The extent of MEK inhibition correlated with the extent of loss of ERK1/2 phosphorylation and induction of $1 \mathrm{Bcl}-2$ family member, the proapoptotic $\mathrm{BH} 3$-only protein Bim.

The inactivation of ERK $1 / 2$ and induction of Bim were accompanied by a decrease in the apparent molecular weight of Bim, which was indicative of dephosphorylation, confirmed by $\lambda$ phosphatase analysis. Because ERK1/2 can phosphorylate Bim, thereby priming it for ubiquitination and proteasomal degradation (25-27), shutdown of this signaling pathway is likely to account for a major part of the accumulation of Bim. In agreement with this notion, the levels of ERK1/2 phosphorylation correlated inversely with the amount of Bim in our panel of $4 B-R A F$ mutant tumors (Figure 4C) and also in a selection of other cell lines $\left(r^{2}=0.4495\right.$; Supplemental Figure 8$)$. Furthermore, it was recently demonstrated that ERK1/2-mediated phosphorylation of $\mathrm{Bim}_{\mathrm{EL}}$ can also promote its rapid dissociation from prosurvival Bcl-2 family members (28). We expect that MEK inhibitorinduced shutdown of this ERK1/2-mediated process promotes apoptosis in $B-R A F$ mutant cells by facilitating the binding of Bim $_{\mathrm{EL}}$ to prosurvival Bcl-2 family members.

Experiments using RNAi demonstrated that Bim was essential for MEK inhibition-induced killing and loss of clonogenic poten- 
A

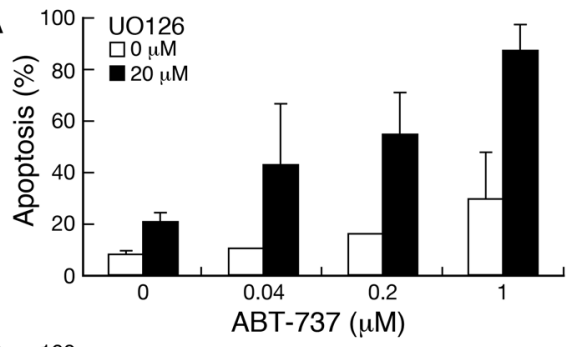

B
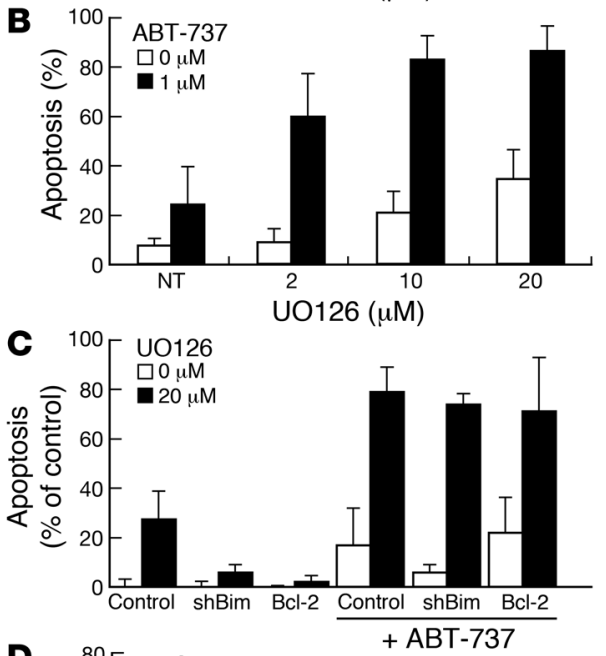

D
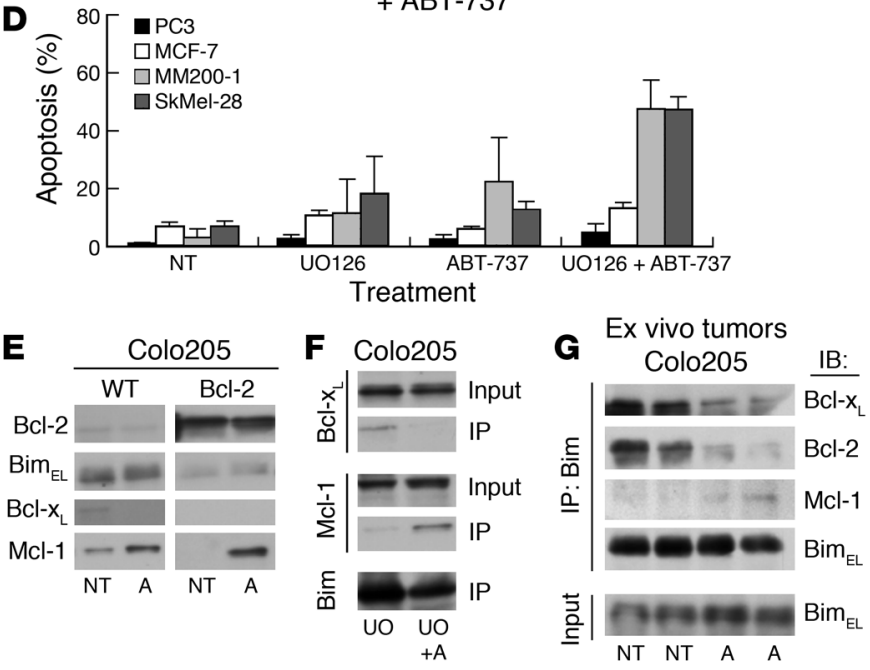

tial of B-RAF mutant tumor cells. The level of protection afforded by Bim KD was comparable to that afforded by Bcl-2 overexpression at early time points, but it was considerably less efficient after more protracted MEK inhibition. This is likely the result of incomplete Bim KD, but it is also possible that activation of other BH3only proteins or inactivation of prosurvival Bcl-2 family members contributed to MEK-induced tumor cell killing, although we saw no evidence in support of this possibility.

Apoptosis induction is thought to require antagonism of all prosurvival Bcl-2 family members expressed in a particular cell by BH3-only proteins (18). We found that the B-RAF mutant tumor cells that were most resistant to MEK inhibitor-induced apoptosis expressed the lowest levels of Bim and the highest levels of Bcl-2. Therefore, inefficient tumor cell killing is probably a consequence of incomplete neutralization of Bcl-2.

\section{Figure 5}

Addition of ABT-737 greatly enhances the MEK inhibition-induced apoptosis of $B-R A F$ mutant tumor cells by increasing the binding of Bim to $\mathrm{Mcl}-1$. (A and B) Colo205 cells were treated with ABT737 plus 0 or $20 \mu \mathrm{M}$ U0126 (A) or with U0126 plus 0 or $1 \mu \mathrm{M}$ ABT-737 (B). Cell killing was assessed after $48 \mathrm{~h}$ as described in Figure 3B. (C) Bim RNAi KD or Bcl-2-overexpressing Colo205 cells were treated with 0 or $1 \mu \mathrm{M}$ ABT-737 plus 0 or $20 \mu \mathrm{M}$ UO126, and cell killing was assessed after $48 \mathrm{~h}$. Data show percent apoptosis compared with untreated cells. (D) MM200-1, SkMel-28, PC3, or MCF-7 cells were not treated or were treated with $20 \mu \mathrm{M}$ U0126, $1 \mu \mathrm{M}$ ABT-737, or both, and cell killing was assessed after $48 \mathrm{~h}$. For A-D, data represent mean \pm SD of 3 independent experiments. (E) Colo205 or Colo205-Bcl-2 cells were not treated or were treated for $18 \mathrm{~h}$ with $1 \mu \mathrm{M} \mathrm{ABT-737}(\mathrm{A})$, and lysates were subjected to antiBim immunoprecipitation and Western blot analysis. (F) Colo205 cells were treated for $18 \mathrm{~h}$ with $20 \mu \mathrm{M}$ UO126 (UO) in the presence or absence of $1 \mu \mathrm{M} \mathrm{ABT-737.} \mathrm{Lysates} \mathrm{were} \mathrm{subjected} \mathrm{to} \mathrm{anti-Bim}$ immunoprecipitation and Western blot analysis. (G) CBA nu/nu mice were inoculated with Colo205 tumor cells; when tumors were palpable, mice were treated with $75 \mathrm{mg} / \mathrm{kg}$ ABT-737 daily for $2 \mathrm{~d}$. Tumors were dissected $48 \mathrm{~h}$ later, and lysates were subjected to anti-Bim immunoprecipitation and Western blotting.

We and others have previously shown that $\mathrm{BH} 3$ mimetics can potently collaborate with the EGF receptor tyrosine kinase inhibitor gefitinib (12-14), and the BCR-ABL tyrosine kinase inhibitor imatinib (11), in the treatment of tumor cells transformed by these oncogenic kinases. Shutdown of the MEK-ERK1/2 pathway was found to be critical for imatinib-induced (11) as well as gefitinib-induced (12-14) tumor cell killing. Accordingly, in the present study we found that ABT-737 synergized with MEK inhibition in the killing of $B-R A F$ mutant tumor cells, even those that spontaneously or through experimental modification expressed abnormally high levels of $\mathrm{Bcl}-2$ or low levels of Bim.

Previous work (9) showed that MEK inhibitors could cause growth arrest, although not substantial regression, of xenografted $B-R A F$ mutant tumors in nude mice. Here, we found that the MEK inhibitor PD0325901 synergized with ABT-737 in vivo to cause prolonged regression of $B-R A F$ mutant tumors in nude mice. The tumor growth delay achieved with combination treatment was highly significant compared with the effects observed with PD0325901 alone. Notably, these results were achieved with low doses of PD0325901, which produced minor growth-inhibitory effects when applied alone. In addition, tumors remained susceptible to retreatment with PD0325901 and, even more impressively, to retreatment with the combination of PD0325901 and ABT-737 at the time of tumor relapse, which indicates that extended treatment regimens may be even more efficacious.

Others have proposed that dephosphorylation of $\mathrm{Bcl}-2$ is critical for the synergistic interaction between MEK inhibition and ABT737 in the killing of acute myeloid leukemia cells (29). This appears unlikely in Colo205 cells, since they express only very low levels of Bcl-2 (Figure 4C). Instead, we believe that ABT-737 liberates Bim (induced by MEK inhibition) and possibly other BH3-only proteins from Bcl-2 and Bcl- $x_{\mathrm{L}}$, thereby allowing efficient neutralization of all prosurvival Bcl-2 family members, including Mcl-1 and potentially $\mathrm{A} 1$, in the tumor cells.

Collectively, studies with tumor cells addicted to 3 different oncogenic kinases - BCR-ABL (11), mutated EGF-R (12-14), and now mutated $B-R A F$ - demonstrate that their killing by specific 

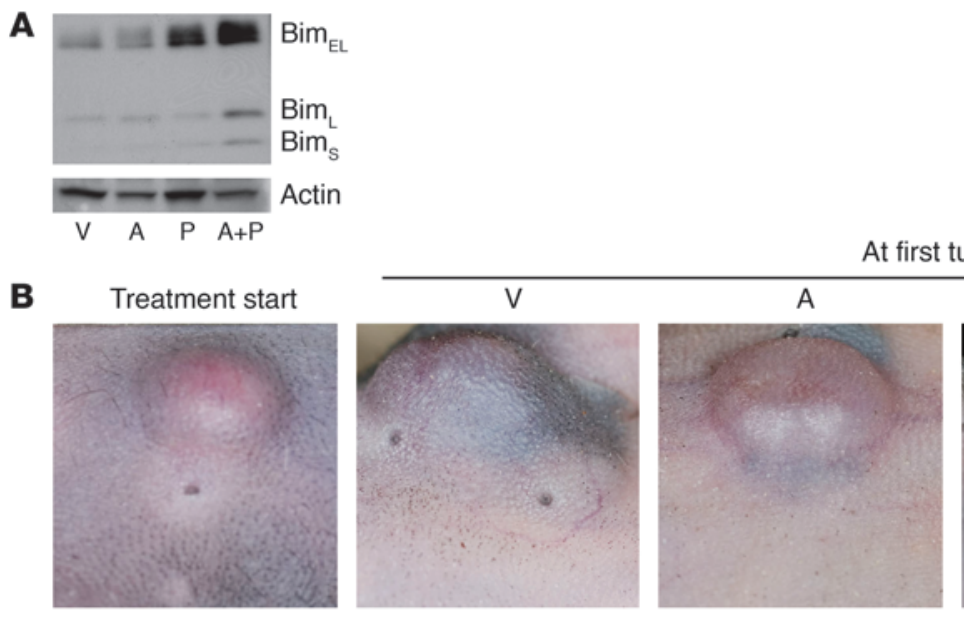

At first tumor cull

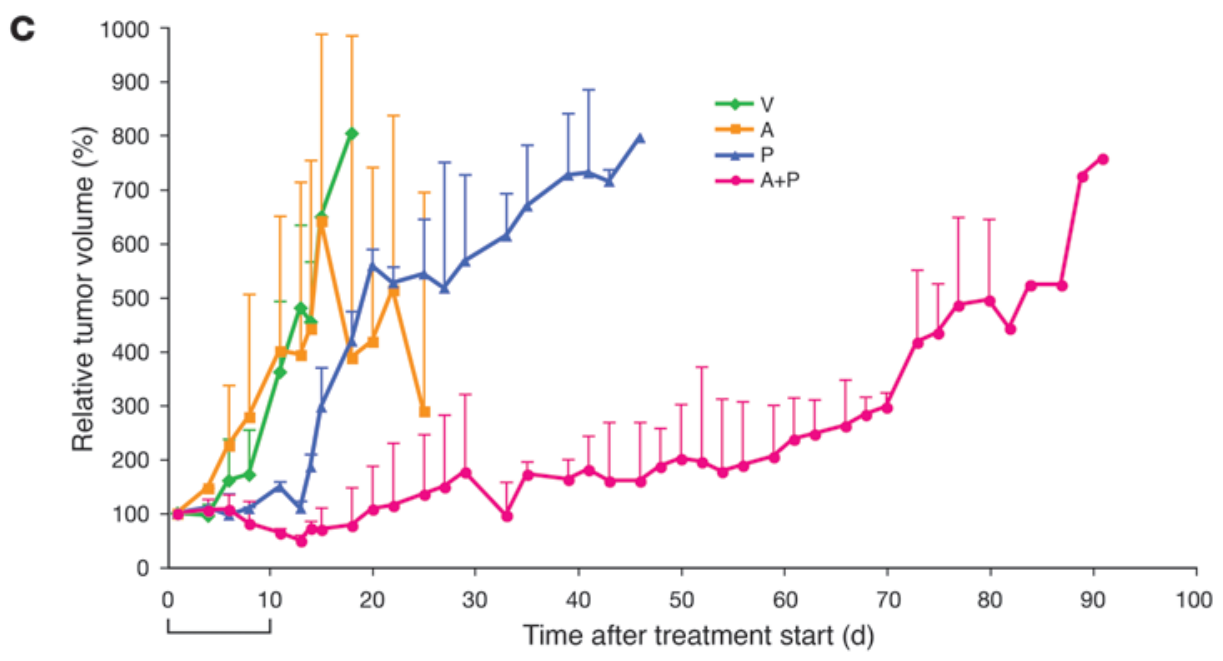

\section{Figure 6}

ABT-737 enhanced the therapeutic effect of PD0325901 in the treatment of $B$ - $R A F$ mutant tumor-bearing nude mice. (A) CBA nu/nu mice were inoculated with SkMel-28 tumor cells; when tumors reached the target size of $0.3 \mathrm{~cm}^{3}$, mice were treated with PD0325901 (P; $3 \mathrm{mg} / \mathrm{kg}$ body weight), ABT-737 (75 mg/kg body weight), both drugs $(A+P)$, or vehicle $(V)$ daily for $2 \mathrm{~d}$. Tumors were then dissected, and cell lysates were subjected to Western blot analysis with antibodies to Bim. (B and C) SkMel-28 tumor cells were inoculated into CBA nu/nu mice; once tumors reached the target size of $0.1 \mathrm{~cm}^{3}$, mice were treated once daily for 10 consecutive $d$ with PD0325901, ABT-737, both drugs, or vehicle. (B) Representative tumors from $\mathbf{C}$ at the time of first treatment and at time of cull of the first tumor-bearing mice (vehicle treated). (C) Average tumor size, measured throughout and represented as the percentage of tumor size at the time treatment began. $n=10-12$ mice per treatment group. Data are mean \pm SD.

kinase inhibitors requires upregulation and dephosphorylation of Bim. A critical common component of the action of these drugs - imatinib, gefitinib, erlotinib, and MEK inhibitors - is the inhibition of ERK1/2, which is known to regulate the proapoptotic activity and level of Bim (17). However, it is not clear why inhibition of MEK, and consequently ERK1/2, is not as potent in killing cells bearing RAS mutations. It may be that oncogenic RAS exerts prosurvival functions in addition to ERK1/2-mediated suppression of Bim that need to be antagonized in order to achieve efficient tumor cell killing. It is well known that Ras is able to activate a number of cellular targets, such as PI3K, Ras GDS, and Tiam-1 (reviewed in ref. 30), some of which have established roles in driving tumorigenesis and preventing apoptosis. Perhaps the most obvious candidate is PI3K, which is known to activate the prosurvival AKT pathway (31), providing a straightforward explanation for the additional prosurvival capacity of Ras.
In conclusion, our results demonstrate that Bim is essential for MEK inhibition-induced killing of $B-R A F$ mutant tumors and that addition of ABT-737 can overcome resistance of tumor cells to MEK inhibitors associated with low levels of Bim or high levels of $\mathrm{Bcl}-2$. These in vivo data suggest that the combination of a MEK inhibitor and a BH3 mimetic could be a powerful new approach in the clinic for treating patients with tumors harboring $B-R A F$ mutations, such as melanomas, which are commonly profoundly resistant to anticancer therapy (32).

\section{Methods}

Cell lines, expression constructs, and cell transfection. Colo205 and HT-29 are cell lines derived from a colorectal tumor; PC3 is a prostate cancer cell line; MCF-7 is a breast cancer line; Ramos, Raji, and SU-DH-L4 are lymphoma cell lines; H1650 is a non-small cell lung cancer cell line; SkMel-28, MM200-1, A375, G361, UACC62, SkMel-31, and Mel-RMU are all melanoma 
cell lines. Melanoma cell lines were provided by G. Boyle (Queensland Institute of Medical Research, Brisbane, Queensland, Australia), P. Hersey (University of Newcastle, Newcastle, New South Wales, Australia), and J. Blaydes (University of Southampton, Southampton, United Kingdom) and were maintained in RPMI-1640 containing HEPES and 10\% heatinactivated FCS (JRH Biosciences), with passaging through trypsinization. QVD-OPH (MP Biomedicals) was used in experiments at a final concentration of $25 \mu \mathrm{M}$ and was added to cells $30 \mathrm{~min}$ prior to the addition of MEK inhibitors. The inhibitors UO126, PD98059 (both from CST), SP6, LY294002 (Merck), ABT-737 (provided by J. Baell, WEHI), and PD0325901 were all dissolved in DMSO and used at the concentrations indicated.

Expression constructs for human FLAG-tagged Bcl-2 (33), the anti-Bim shRNA (34), and a control scrambled shRNA construct (12) were described previously. The independent shRNA to human Bim and nonsilencing control shRNAs were gifts of the Victorian Centre for Functional Genomics.

Transfection with Fugene (Roche) was performed according to the manufacturer's instructions. Transfected cells were selected with $1 \mu \mathrm{g} / \mathrm{ml}$ puromycin and single-cell cloned by limiting dilution. FLAG-tagged proteins were detected by cytoplasmic immunofluorescence staining with anti-FLAG antibody (M2; Sigma-Aldrich) and flow cytometric analysis in a FACScan (BD).

Western blotting. Protein samples were separated by SDS-PAGE and then blotted onto PVDF membranes (Hybond P; Amersham Biosciences). The membranes were blocked with $5 \%$ nonfat dry milk in PBS with $0.1 \%$ Tween 20 (Sigma-Aldrich) and then probed with antibodies against Bcl-w (13F9; Alexis), Bcl- $\mathrm{x}_{\mathrm{L}}$ (BD Biosciences), Bim (clone 3C5; Alexis; or polyclonal Ab; Stressgen), Bad (Stressgen), phosphorylated Bad (Ser $\left.{ }^{112}\right)$, phosphorylated Bad $\left(\operatorname{Ser}^{136}\right)$, Bax (N20; Santa Cruz Biotechnology Inc.), Bak (SigmaAldrich), cleaved caspase-3, phosphorylated ERK1/2 (Thr $\left.{ }^{202} / \mathrm{Tyr}^{204}\right)$, total ERK1/2, phosphorylated Akt (193H12, Ser ${ }^{473}$ ), total Akt (all from CST), Bax (Upstate), human Bmf (Alexis), heat shock protein 70 (Hsp70, N6; gift of R. Anderson, Peter MacCallum Cancer Centre, Melbourne, Victoria, Australia), Mcl-1 (Dako), PARP (Alexis), Puma (NT; Pro-Sci), or $\beta$-actin (Sigma-Aldrich). Detection was performed with HRP-conjugated secondary antibodies and ECL (Amersham Biosciences).

Immunoprecipitation. For immunoprecipitation, cell lysates were first precleared by incubation for $2 \mathrm{~h}$ with protein $\mathrm{G}$-sepharose (Amersham Biosciences) and then incubated for $2 \mathrm{~h}$ with anti-Bim (3C5) mAb-coupled sepharose beads. Beads were then washed before elution with $0.1 \mathrm{M}$ glycine $\mathrm{HCl}$ ( $\mathrm{pH}$ 2.7) followed by neutralization and boiling in loading buffer.

Cell death assays. Cell death was assessed by flow cytometric analysis following release of the cells from the culture dish through trypsinization, either by staining with propidium iodide plus FITC-conjugated annexin $V$ or by measuring the percentage of cells that underwent DNA fragmentation (sub-G $\mathrm{G}_{1} \mathrm{DNA}$ ), as detailed previously (35). To compare between transfectants, we expressed apoptosis as percent of control, calculated as $(1$ - [100 - sample] $) /(100$ - control). The latter technique was also used to assess changes in cell cycle distribution. For clonogenic survival assays, cells were seeded at $2.0 \times 10^{5}$ cells $/ \mathrm{ml}$ and treated for 24 or $48 \mathrm{~h}$ with $20 \mu \mathrm{M}$ UO126 (CST) in the presence or absence of ABT-737 (1 $\mu \mathrm{M}$ unless otherwise indicated). Cells were then trypsinized and washed to remove drugs before adding fresh medium and seeded at different cell densities (1-2,400 cells/well with at least 8 wells per cell density and treatment) in 96-well plates (Nunc). Clonogenicity was examined by counting the number of wells with colonies after 10-12 d of culture and performing linear regression analysis as previously reported (36).

Animal studies. Athymic CBA $n u / n u$ mice (6-8 wk old; WEHI animal breeding facility) were used for animal studies with approval from the Melbourne Health Animal Ethics Committee and the WEHI Animal Ethics Committee. Tumors were generated in mice by subcutaneous injection of $5 \times 10^{6}$ Colo2 05 or SkMel-28 tumor cells together with $10 \%$ (final $v / v$ ) Matrigel (BD Biosciences - Pharmingen). Tumor growth was monitored by measuring 2 perpendicular axes using calipers. After tumors had grown to the approved size $\left(0.1 \mathrm{~cm}^{3}\right.$ for SkMel-28 or $0.2 \mathrm{~cm}^{3}$ for Colo205, calculated as $\pi / 6 \times$ [larger diameter $\times$ smaller diameter $\left.{ }^{2}\right]$ ), mice were randomized into 4 treatment groups: $3 \mathrm{mg} / \mathrm{kg}$ PD0325901 by oral gavage, $75 \mathrm{mg} / \mathrm{kg}$ ABT-737 intraperitoneally, both drugs, or vehicle only as a control. For tumor-bearing mice that were to be harvested $48 \mathrm{~h}$ after treatment, the tumors were allowed to grow to $0.3 \mathrm{~cm}^{3}$ prior to treatment.

PD0325901 was formulated in $0.5 \%$ hydroxypropyl methylcellulose plus $0.2 \%$ Tween 80 and administered by oral gavage. ABT-737 was formulated as described previously (21) and injected intraperitoneally. Drugs were administered daily for $10 \mathrm{~d}$, and tumor size was measured every $2-3 \mathrm{~d}$. Mice were sacrificed when the tumors reached the target volume $\left(0.5 \mathrm{~cm}^{3}\right.$ for SkMel-28 or $0.7 \mathrm{~cm}^{3}$ for Colo205). Mice were weighed daily during therapy (days 1-10) and also if appearing unwell and at cull. No mice developed a significant change in weight (loss of more than $10 \%$ total body weight). Mice were bled for hematologic analysis at $16 \mathrm{~h}, 48 \mathrm{~h}$, and $11 \mathrm{~d}$ as well as at cull. For a subset of mice, when tumors reached the predetermined end point (SkMel-28, $0.5 \mathrm{~cm}^{3}$; Colo205, $0.7 \mathrm{~cm}^{3}$ ), mice were retreated for a further $10 \mathrm{~d}$ with the same treatment regimen and/or were euthanized when tumors reached $0.8 \mathrm{~cm}^{3}$.

For biochemical analyses, tumors were dissected, prepared as a singlecell suspension, and snap frozen for lysis and subsequent assessment by Western blotting or immunoprecipitation. Effects on tumor growth delay were assessed by determining percent ILS, calculated as the ratio of median times for tumors to reach predetermined size $x$ in treated/ control groups (26).

Statistics. Prism software (version 5.0; GraphPad Software Inc.) was used for generating Kaplan-Meier statistical analysis (using a log-rank test) of survival of mice related to tumor size end point. $P$ values of less than 0.05 were considered significant.

\section{Acknowledgments}

We are grateful to G. Boyle, P. Hersey, and J. Blaydes for the kind gift of cell lines, J. Baell for ABT-737, and R. Anderson for antibodies. We thank all of our colleagues in the Molecular Genetics of Cancer Division and Cancer Sciences Division of Southampton University for gifts of reagents, scientific advice, and technical support. This work was supported by the National Health and Medical Research Council (NHMRC; program no. 257502; RD Wright Biomedical CDA 406675), by a grant from the Leukemia and Lymphoma Society (SCOR grant no. 7015), by National Cancer Institute, NIH, grants CA 80188 and CA 43540, and by a Leukemia Research UK fellowship (to M.S. Cragg).

Received for publication February 25, 2008, and accepted in revised form September 3, 2008.

Address correspondence to: Andreas Strasser, The Walter and Eliza Hall Institute of Medical Research, 1G Royal Parade, Parkville, 3050 Victoria, Australia. Phone: 61-3-9345-2624; Fax: 61-3-93470852; E-mail: strasser@wehi.edu.au. Or to: Mark S. Cragg, Cancer Sciences Division, Southampton University School of Medicine, Tremona Road, Southampton General Hospital, Southampton SO16 6YD, United Kingdom. Phone: 44-2380-777222 ext. 8056; Fax: 44-2380-704061; E-mail: msc@soton.ac.uk.

Mark S. Cragg, Andreas Strasser, and Clare L. Scott are co-senior authors. 
1. Peyssonnaux, C., and Eychene, A. 2001. The Raf/ MEK/ERK pathway: new concepts of activation. Biol. Cell. 93:53-62.

2. Cohen, C., et al. 2002. Mitogen-actived protein kinase activation is an early event in melanoma progression. Clin. Cancer Res. 8:3728-3733.

3. Brose, M.S., et al. 2002. BRAF and RAS mutations in human lung cancer and melanoma. Cancer Res. 62:6997-7000.

4. Cohen, Y., et al. 2003. BRAF mutation in papillary thyroid carcinoma. J. Natl. Cancer Inst. 95:625-627.

5. Rajagopalan, H., et al. 2002. Tumorigenesis: RAF/ RAS oncogenes and mismatch-repair status. Nature. 418:934.

6. Davies, H., et al. 2002. Mutations of the BRAF gene in human cancer. Nature. 417:949-954.

7. Bos, J.L. 1989. ras oncogenes in human cancer: a review. Cancer Res. 49:4682-4689.

8. Pavey, S., et al. 2004. Microarray expression profiling in melanoma reveals a BRAF mutation signature. Oncogene. 23:4060-4067.

9. Solit, D.B., et al. 2006. BRAF mutation predicts sensitivity to MEK inhibition. Nature. 439:358-362.

10. Strasser, A., O'Connor, L., and Dixit, V.M. 2000. Apoptosis signaling. Annu. Rev. Biochem. 69:217-245.

11. Kuroda, J., et al. 2006. Bim and Bad mediate imatinib-induced killing of $\mathrm{Bcr} / \mathrm{Abl}+$ leukemic cells, and resistance due to their loss is overcome by a BH3 mimetic. Proc. Natl. Acad. Sci. U. S. A. 103:14907-14912.

12. Cragg, M.S., Kuroda, J., Puthalakath, H., Huang, D.C., and Strasser, A. 2007. Gefitinib-induced killing of NSCLC cell lines expressing mutant EGFR requires BIM and can be enhanced by BH3 mimetics. PLoS Med. 4:1681-1689; discussion 1690.

13. Gong, Y., et al. 2007. Induction of BIM is essential for apoptosis triggered by egfr kinase inhibitors in mutant EGFR-dependent lung adenocarcinomas. PLoS Med. 4:e294.

14. Costa, D.B., et al. 2007. BIM mediates EGFR tyro- sine kinase inhibitor-induced apoptosis in lung cancers with oncogenic EGFR mutations. PLoS Med. 4:1669-1679; discussion 1680.

15. O'Connor, L., et al. 1998. Bim: a novel member of the Bcl-2 family that promotes apoptosis. $E M B O J$. 17:384-395.

16. Puthalakath, H., and Strasser, A. 2002. Keeping killers on a tight leash: transcriptional and posttranslational control of the pro-apoptotic activity of BH3-only proteins. Cell Death Differ. 9:505-512.

17. Ley, R., Ewings, K.E., Hadfield, K., and Cook, S.J. 2005. Regulatory phosphorylation of Bim: sorting out the ERK from the JNK. Cell Death Differ. 12:1008-1014.

18. Willis, S.N., et al. 2007. Apoptosis initiated when $\mathrm{BH} 3$ ligands engage multiple Bcl-2 homologs, not Bax or Bak. Science. 315:856-859.

19. Chen, L., et al. 2005. Differential targeting of prosurvival Bcl-2 proteins by their $\mathrm{BH} 3$-only ligands allows complementary apoptotic function. Mol. Cell. 17:393-403.

20. Kuwana, T., et al. 2005. BH3 Domains of BH3Only proteins differentially regulate Bax-mediated mitochondrial membrane permeabilization both directly and indirectly. Mol. Cell. 17:525-535.

21. Oltersdorf, T., et al. 2005. An inhibitor of Bcl-2 family proteins induces regression of solid tumours. Nature. 435:677-681.

22. Sebolt-Leopold, J.S., and Herrera, R. 2004. Targeting the mitogen-activated protein kinase cascade to treat cancer. Nat. Rev. Cancer. 4:937-947.

23. Mason, K.D., et al. 2007. Programmed anuclear cell death delimits platelet life span. Cell. 128:1173-1186.

24. Shoemaker, A.R., et al. 2006. A small-molecule inhibitor of Bcl-XL potentiates the activity of cytotoxic drugs in vitro and in vivo. Cancer Res. 66:8731-8739.

25. Akiyama, T., et al. 2003. Regulation of osteoclast apoptosis by ubiquitylation of proapoptotic BH3-only Bcl-2 family member Bim. EMBO J. 22:6653-6664.
26. Ley, R., Balmanno, K., Hadfield, K., Weston, C., and Cook, S.J. 2003. Activation of the ERK1/2 signaling pathway promotes phosphorylation and proteasome-dependent degradation of the BH3-only protein, Bim. J. Biol. Chem. 278:18811-18816.

27. Luciano, F., et al. 2003. Phosphorylation of Bim-EL by Erk1/2 on serine 69 promotes its degradation via the proteasome pathway and regulates its proapoptotic function. Oncogene. 22:6785-6793.

28. Ewings, K.E., et al. 2007. ERK1/2-dependent phosphorylation of BimEL promotes its rapid dissociation from Mcl-1 and Bcl-xL. EMBO J. 26:2856-2867.

29. Konopleva, M., et al. 2006. Mechanisms of apoptosis sensitivity and resistance to the $\mathrm{BH} 3$ mimetic ABT-737 in acute myeloid leukemia. Cancer Cell. 10:375-388.

30. Shaw, R.J., and Cantley, L.C. 2006. Ras, PI(3)K and mTOR signalling controls tumour cell growth. Nature. 441:424-430.

31. Amaravadi, R., and Thompson, C.B. 2005. The survival kinases Akt and Pim as potential pharmacological targets. J. Clin. Invest. 115:2618-2624.

32. Soengas, M.S., and Lowe, S.W. 2003. Apoptosis and melanoma chemoresistance. Oncogene. 22:3138-3151.

33. Huang, D.C., et al. 1999. Activation of Fas by FasL induces apoptosis by a mechanism that cannot be blocked by Bcl-2 or Bcl-x. . Proc. Natl. Acad. Sci. U. S. A. 96:14871-14876.

34. Bouillet, P., Robati, M., Bath, M.L., and Strasser, A. 2005. Polycystic kidney disease prevented by transgenic RNA interference. Cell Death Differ. 12:831-833.

35. Cragg, M.S., et al. 2000. Complement mediated cell death is associated with DNA fragmentation. Cell Death Differ. 7:48-58.

36. Strasser, A., Harris, A.W., Jacks, T., and Cory, S. 1994. DNA damage can induce apoptosis in proliferating lymphoid cells via p53-independent mechanisms inhibitable by Bcl-2. Cell. 79:329-339. 